\title{
Microbiological air analysis of industrial premises and photocatalysis efficiency for its disinfection
}

\author{
V.S. Shkrabak ${ }^{1}$, E.V. Zhgulev ${ }^{1}$, E.I. Gavrikova ${ }^{2}$, and R.V. Shkrabak ${ }^{1}$ \\ ${ }^{1}$ St-Petersburg State Agrarian University, St. Petersburg - Pushkin 196601, Russia \\ ${ }^{2}$ Orel State Agrarian University named after N.V. Parakhin, Orel 302019, Russia
}

\begin{abstract}
This article analyzes the reasons of temporary disability of laborers in Agro Industrial Complex, dealing with microbial content in industrial premises. Development and implementation of equipment for correction of microbial content level results in reduction of general morbidity rate and duration of temporary disability among laborers in Agro Industrial Complex. It will help to increase labor efficiency and decrease labor intensity of production. Biofilm resistance against antibacterial agents is a vital problem during disinfection treatments. Increase in bacteria survival potential and reduction in sensitivity to the substances eliminating them, results in need for increased dosing of the latter decreased efficiency of sanitation. This is a stimulus to develop alternative antibacterial compositions and methods of disinfection treatment. The basic difference of the suggested photodynamic treatment from the disinfection methods with application of antimicrobial preparations is the lack of resistibility to destabilizing oxidant - singlet oxygen. Before disinfection treatment, we determined total microbial air contamination according to the original method, providing a possibility to use air medium sampling of the total volume of indoor area. Experimental results have shown a decrease in total microbial contamination by a factor of 5.5 on average.
\end{abstract}

\section{Introduction}

One of the factors influencing the efficiency of labor potential utilization is sanitary-hygienic state of working environment, particularly microclimate parameters, such as increased microbial content in production premises air space. The increase in microorganisms number and amount of their life activity products results in decrease of laborers systemic immunity leading to proneness growth to infection, diseases, and thus, to extension of temporary disability terms, working hours loss and as a result to reduction of production economic benefits.

Since 1970s, numerous publications connected with the problem of air medium cleaning of cattle-breeding and poultry premises from various adversely active components, have appeared in the national and international scientific and technical literature. The great part of these publications is devoted to investigation of air medium for the presence of dust, microorganisms, hazardous impurities, gases, impact of these parameters on human health and productivity of animals and birds [1-3]. The methods and means of air cleaning from microbial contamination are reflected in numerous investigations [4-6].

Systematical data on microbial content of cattlebreeding and poultry premises and on meat processing enterprises are absent. There are only separate items of information on microbial aerosol composition. It is proved that bacterial contamination of air medium is 7000-280000 units $/ \mathrm{m}^{3}$ and more, which by ten folds exceeds the recommended specified values $(\mathrm{MAC}=$ 7000 units $/ \mathrm{m}^{3}$ ). At that, more than $50 \%$ of bacteria can be pathogenic to humans [7].

However, the hazard level conditioned with the laborers contact with microorganisms for various enterprises of Agro Industrial Complex, is not determined. The emphasis is on the acute and occupational diseases, connected with germs of especially dangerous anthropo-zoogenous infections. At the same time, it is proved that, high number of microorganisms of nonpathogenic and potentially pathogenic forms decreases immune reactivity of organism of laborers and as a result, we have growth of liability to disease with temporary disability.

The decrease of disease incidence of employees working at Agro Industrial Complex enterprises is one of the main tasks of agricultural production. Effective work in this direction has great general economic meaning, because it results in decrease of disease-related labor losses. In this connection, there is a need to develop ways and means of protection from microorganisms for laborers of Agro Industrial Complex.

Bacteria in the environmental conditions most often are united into cenoses - biofilms, which are exceedingly resistant to the external influence because of cell phenotype change and extracellular polymeric substances synthesized by them, forming mechanical base and system protective layer (matrix). Being taken individually, suspended microorganisms (planktonic bacteria) possess the resistance to toxic substances

* Corresponding author: v.shkrabak@mail.ru 
activity, which is determined by the target alteration, exposure retarding and toxins exocytic release, biological membranes failure. Insensibility of biofilm bacteria to the destructive preparations is considerably higher than that of its individual component microorganisms. This is because of the fact that matrix, during the attack of the substances destabilizing it, activates the mechanisms that do not allow the contact of microorganisms, located in the deep biofilm layers, with these substances [8,9]. Matrix is not only a carcass for biofilm but also a filtering element of the intercellular space, penetrated with channels for bacteria life support. Biofilm stabilizing matrix changes dynamically reacting to the environmental exposure, selectively functioning with respect to different types of antibiotics.

More recently requirements to technologies and machinery focused on elimination of adverse scents, contamination of air with emissions increased considerably and accordingly at the present time new technologies to support optimal environmental parameters in working areas are created.

The application of new prospective methods of microorganism disinfection helping to destroy biofilms and eliminate bacteria by means of photocatalytic reactions, permits to avoid the drawbacks of common methods of decontamination of microorganisms [10]. Chemical reactions on the photocatalyst surface run much faster in case of absorption of photon beam with this surface. To destroy volatile organic compounds and reduce the number of microorganisms in the air, a powerful oxidation agent - oxygen radicals - is used, which is obtained because of the interaction of photocatalyst and UV-radiation.

\section{Results and discussion}

In the microbiological practices, the most widespread method of determination for air microbial content of indoor areas is calculation of microorganism colonies grown after incubation, free deposited from air on the nutrient medium of open Petri dishes. According to the Osmelyansky's formula, the number of bacteria sedimented on $100 \mathrm{sm}^{2}$ on open Petri dishes during 5 minutes, coincides with the number of bacteria in 101 of the analyzed air. However, the test results can be argued in connection with localized sampling, and with the fact that small size and weight do not allow some bacteria getting into Petri dish.

The possibility of air medium sampling of all indoor area volume allows increasing reliability of estimate of air microbial content. In the method developed by us, this result is achieved by counting bacteria, sedimented on the filter paper, placed in the air filter casing of the ventilation system.

For proper filter paper fastening we developed a design of the air filter [1], used in the ventilation system of a building.

Considering that, the main part of bacteria is absorbed with the airborne dust; the methods aimed at reducing the bacterial content by means of dust elimination have acquired wide application. Filtering elements have different fixations in the devices: at variable or small flow of air the filter cloth typically produced from fabric, is placed in a frame, fixed by means of latches or thread joints.

However, in case of dust elimination of heavily contaminated airflows, large-size filters are produced with an increased safety factor. The form of the replaceable filters is presented with various frames with filter material fixed on them.

Frame configuration is performed with consideration of aerodynamic loadings and is made suitable to the device in a transverse direction relatively the air flow in the ventilation systems, though for most cases a filter element together with a frame are one-time elements. In comparison with common devices [2] the suggested construction is comfortable in application and allows secure fastening of the filter cloth.

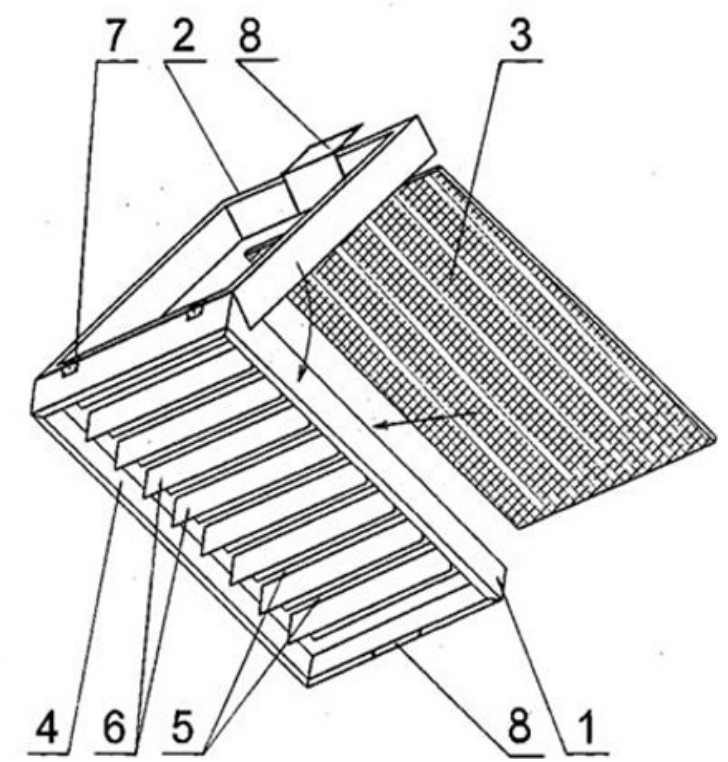

Fig. 1. Air filter casing: 1 - Internal frame; 2 - External frame; 3 - Filter cloth; 4 - Lateral faces; 5 - Clamp bars; 6 - Guiding elements; 7 - Pivot hinges; 8 - Magnetic holders

The basic elements of the air filter casing (fig.1) are internal frame 1 , internal frame 2 and filter cloth placed between them 3. A one-piece rectangular metal plate forms a base for the internal frame 1, herewith the plate folded edges form lateral faces 4 .

Depending on the air direction, the angular position of guiding elements 6 varies; narrow clamp bars 5 change into the guiding elements. Magnetic holders 8 are located opposite pivot hinges 7 on both frames correspondingly. Magnetic holder 8 of external frame 2 is produced in the form of an angle, one edge of which has a flange.

The total dimensions of the air filter casing are chosen according to the section of the system air pipe. The air filter must be mounted into the ventilation device considering that airflow should come from the side of external frame 2 .

In order to change the filter cloth, it is necessary to withdraw the air filter casing from the air pipe, open it holding on the angle flange of magnetic holder 8 , 
withdraw the used filter cloth 3 , insert new filter cloth in internal frame 1 , close external frame 2 .

The suggested construction permits the use of any filter material, without application of one-time means to provide rigidity of its edges. The pivot hinges and the magnetic holders with the angles reduce considerably the time for filter cloth change. Transverse clamp bars prevent deformation and tension of cloth, guiding elements serve for improving aerodynamic qualities of the filter and the airflow organization in the necessary direction.

For our tests between filter cloth 3 and clamp bars 5 we place the narrow strip of the filter paper, whose width $(0.02 \mathrm{~m})$ does not create obstacles for the filter functioning, and the length is chosen according to its dimensions.

By trial and error, we determined that the optimal test duration is 4 hours (fig. 2). Meanwhile, the bulk of indoors airborne microorganisms sediments on the filter paper. Additional exposure is inadvisable because it results only in slight growth of the sedimented microorganisms number. In addition, in the long run, the filtration area decreases, because dust particles obstruct through pores of filter paper.

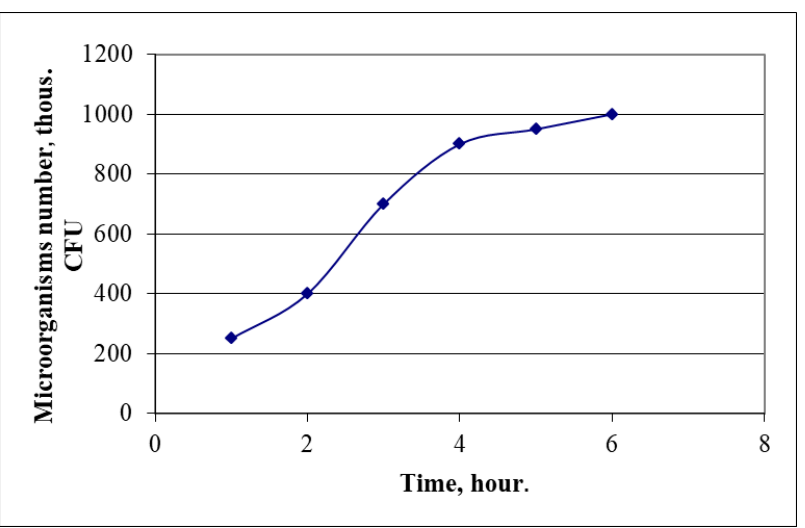

Fig. 2. Dependency diagram of microbial population, sedimented on filter paper from test time

The filter paper strip after extraction from the air filter casing is cut into segments with the length of 0.04 $\mathrm{m}$ for convenience of placing the obtained samples in vials, filled with $10 \mathrm{ml}$ of $1 \%$ sterile solution of glucose on physiological solution. Then, vials with samples are placed into a thermostat at the temperature of $37 \pm 1{ }^{\circ} \mathrm{C}$ for 2 hours. Electro conductivity of solution is recorded with sensor KDS-1038 (fig. 3).

The more microbial population the lower electric conductivity value. Experimentally we determined which value of the solution electric conductivity corresponded to the value of maximum acceptable concentration of microbial aerosol in cattle-breeding premises and made decision on the necessity of disinfection treatment.

For air disinfection treatment of the working zones of the indoor areas, antimicrobial photocatalytic disinfection is used increasingly [13, 14]. Fig. 4 presents the suggested device for photocatalytic air handling.

The device consists of biosensor 1; control appliance 2, containing resistors R1 (3), R2 (4), R3 (5), relay K1 (7), transistors V1 (6), V2 (8), power-supply unit 9, contacts K1.1 (10) relay K1 (7); ac generators 11, 12, casing 13, which contains UV source 14, removable photocatalytic element 15 , electrode 17 in spiral form; electrode 16 in the form of a hollow cylinder 16, located on the external wall of casing 13. Removable photocatalytic element 15 , located on the internal wall of casing 13 , contains a carrier-base, where a photocatalyst is positioned. Electrode 17, produced in the spiral form presses with its winds photocatalytic element 15 to the internal wall of casing 13. Resistors R1 (3), R2 (4) serve to generate shift voltage on the base of transistor V1 (6), necessary for steady operation of control appliance 2 . Resistor R3 (5) is required to limit the current coming via transistor V2 (8).

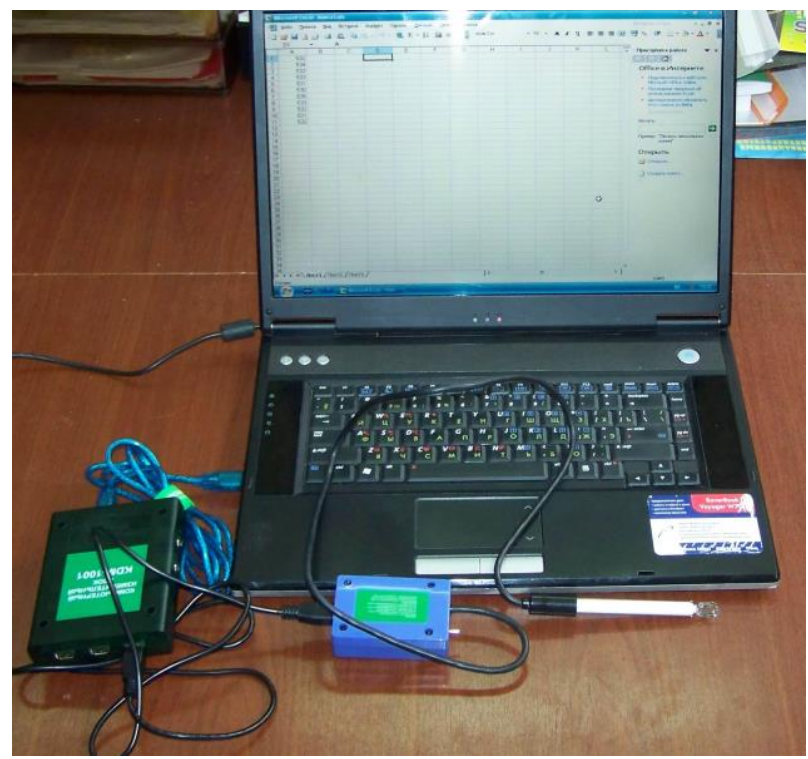

Fig. 3. Sensor KDS-1038

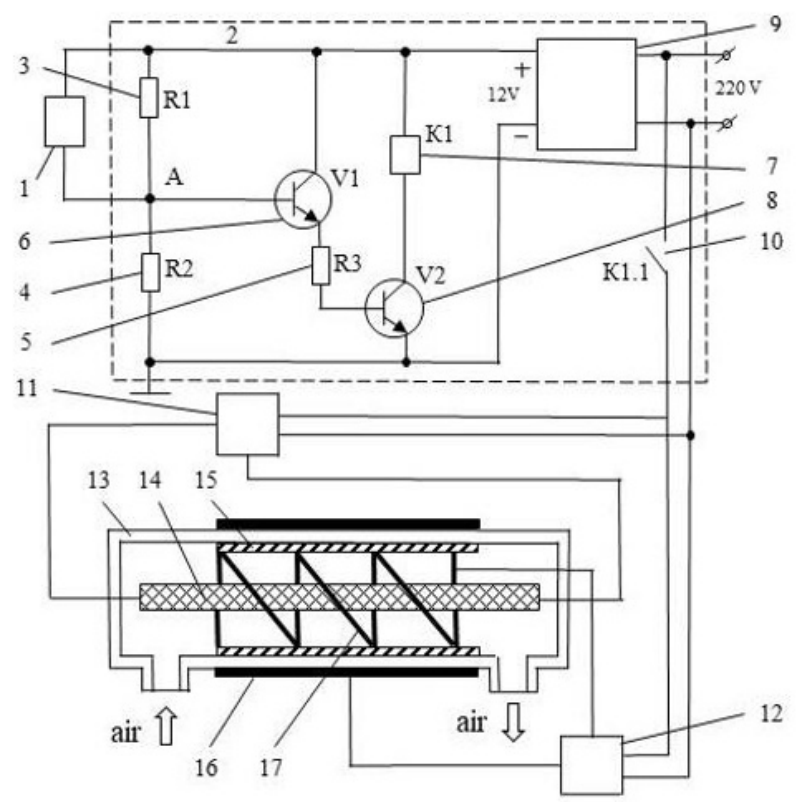

Fig. 4. Device for photocatalytic air handling: 1 - Biosensor; 2 - Control appliance, containing 3, 4, 5 - Resistors, 7- Relay, 6, 8 - Transistors, 9 - Power-supply unit, 10 - Relay contacts 7; 11, 12 - AC generators, 13 - Casing, 14 - UV source, 15 Removable photocatalytic element, 16,17 - Electrodes 
The suggested device operates in the following way.

By means of biosensor 1 the fact of microorganisms presence in the analyzed air is detected, then the signal is issued to control appliance 2 .

In the initial state, the voltage at point $A$, on the base of transistor V1 (6), is such that it is closed. During signal supply (having earth positive potential), in case of excess of maximum acceptable concentration of microorganisms, to input $\mathrm{A}$, the voltage on the base of transistor V1 (6) increases. It opens the transistor. The current flowing via transistor V1 (6) and resistor R3 (5) opens the transistor V2 (8), which in its turn starts transmitting the current sufficient for relay K1 (7) switching on, which with its contacts K1.1 (10) switches AC generators 11 and 12, controlling UV-source 14 and electrodes 16, 17.

Ventilator (not presented in the figure) makes the handled air circulate between input and output of casing 13. Generator 11 feeds UV-source 14, which generates UV-radiation on the internal side of photocatalytic element 15, which causes the photocatalytic reaction. Simultaneously, generator 12 creates the electric potential difference between electrodes 16 and 17 . Thus, cold surface plasma is formed opposite the internal side of photocatalytic element 15 round winds of electrode 17. The surface plasma generates ionic wind for homogenization of the handled air, which increases photocatalysis efficiency.

The suggested device of photocatalytic air handling permits to determine the excessive bacteria content in the air and immediately eliminate them. Disinfection efficiency is connected with combined effect of photocatalysis, taking place at activation of photocatalyst by UV-radiation, and surface plasma. This combination increases the speed of bactericidal treatment and can be used to destroy organic pollutants present in the air.

Air bacterial content were recorded before and after disinfection. During the process of photocatalytic treatment, the decrease of total microbial content was registered (table 1$)$.

Table 1. Air bacterial content at using the photocatalytic disinfection (Total Microbial Count, $\mathrm{CFU} / \mathrm{m}^{3}$ )

\begin{tabular}{|c|c|c|c|c|}
\hline & \multicolumn{4}{|c|}{ Sampling interval } \\
\hline & $\begin{array}{c}\text { Before } \\
\text { treatment }\end{array}$ & in 1 day & on the 8-th day & $\begin{array}{c}\text { on the 15-th } \\
\text { day }\end{array}$ \\
\hline Control & 410 & 431 & 450 & 455 \\
\hline Test & 410 & 312 & 105 & 71 \\
\hline
\end{tabular}

According to the test results, total microbial content decreases by a factor of 5.5 on average

\section{Conclusion}

The efficiency of utilization of farm hand labor potential is determined in large part with the level of work safety conditions in agricultural enterprises. Considering that the low level of technical and technological support of production can considerably determine unfavorable hygienic room indexes, the equipment manufacturing to support the optimal parameters of technological environment is a vital problem.

Sampling of air medium from the whole volume of the indoor area at the account of determination of microbial population sedimented on the filter paper of the ventilation system permitted to increase the reliability of estimate of microbial air content. The application of the suggested technical solution of photocatalytic disinfection allows to decrease the level of microbial content in the treated premises by a factor of 5.5 on average.

Based on the results of the carried out investigations, it is possible to stress, that the complex approach including the determination of general microbial content according to the original method and disinfection by means of the proposed device for photocalytic air treatment, permits to apply the development data into the action plan on health preservation of laborers of Agro Industrial Complex in the conditions of the increased microbial contamination.

\section{References}

1. V.S. Shkrabak, A.V. Lukovnikov, A.K. Turgiev, Life protection in agricultural enterprise (KolosS, Moscow, 2005)

2. A.P. Kondratov, M.V. Ryabkin, A.V. Platonov, Disinfect. Af. 2, 40-43 (2006)

3. S. A. Taubaev, Bul. of Agric. sc. of Kazakh. 3, 67-69 (1981)

4. K. Hashimoto, H. Irie, A. Fujishima, Japan. J. of Appl. Phys. 44(12), 8269-8285 (2005)

5. M. Gellen, K.W. Hock, Stankbestrijdingsteohnicken vook Stallen in de intensieve veehonderij (Wageningen, 1982)

6. V.S. Shkrabak, E.I. Gavrikova, Improvement of prevention ways of industrial risks, dynamic reduction and liquidation of injuries and morbidity of laborers of Agro Industrial Complex 9-12 (SAU, St. Petersburg, 2017)

7. A.I. Netrusov, E.A. Bonch-Osmolovskaya, V.M. Gorlenko, M.V. Ivanov, Microorganisms ecology (Academia, Moscow, 2004)

8. J.L. Pace, M.E. Rupp, R.G. Finch, CRC Press Taylor\&Francis Gr. 7, 109-153 (2005)

9. I.V. Chebotar', A.N. Mayansky, E.D. Konchakova, A.V. Lazareva, V.P. Chistyakova, Clinic. microbial. of antimicrob. Chem. 14(1), 51-58 (2012)

10. O. Carp, C.L. Huisman, A. Reller, Prog. Solid State Chem. 32, 33-177 (2004)

11. K.S. Laktionov, E.I. Gavrikova, Patent RU 2541768 (2015)

12. E.I. Gavrikova, Patent RU 137937 (2014)

13. I.S. Alekseev, N.I. Miklis, S.S. Klimenkov, Bull. of Vitebsk St. Tech. University 2(23), 91-94 (2012)

14. F. Peti, P.-Z. Vyalle, A. Machuka, K. BatioDyupejra, Z.-M. Tati-Bue, Patent RU 2643560 (2014) 